\title{
Pancreatic cyst-solid mass: tuberculosis
}

\author{
C. Wu, S. Wang, B. Hu \\ Department of Gastroenterology, West China Hospital, Sichuan University, China
}

Question: A 29-year-old Chinese male was admitted to our department with a history of right upper quadrant pain for two months, anorexia, weight loss about $5 \mathrm{~kg}$ and without fever. The pain radiated to the back and it got worst lately. He didn't have a history of tuberculosis. The sclera was mild icteric. Laboratory test results showed total bilirubin level increased to 58 umol/L (normal level $<28 \mathrm{umol} / \mathrm{L}$ ) and tumor markers were normal. Chest X-ray was normal. Abdominal contrastenhanced CT showed a $25 \times 30 \mathrm{~mm}$ cyst-solid mass in the head of pancreas (Figure1. A) and the lesion was mild enhancement in arterial phase. Pancreatic tumor was considered. Endoscopic ultrasonography confirmed the mass with uneven echo in the pancreatic head and the boundary of the mass was not clear (Figure 1. B). The lesion may invade the portal vein. As the patient was young and the operation was very traumatic, the patient refused surgery unless the lesion was proved to be a tumor.

Question: Which of the following choices is the correct diagnose?

A: Pancreatic cancer

B: Pancreatic cystadenoma

C: Pancreatic tuberculosis

D: Intraductal papillary mucinous neopalsms

The correct answer is C. Endoscopic ultrasoundguided fine needle aspiration (EUS-FNA) of the lesion was performed in duodenum (Figure 2. A, Video) and some specimen was caseous (Figure 2. B). Histopathological examination showed no malignant cell. Mycobacterium complex was found in acidfast bacillus smear (Figure 2. C) and the diagnose of pancreatic tuberculosis was confirmed. The patient received 12 months of anti-tuberculosis therapy which contained isoniazid, rifampicin and ethambutol. The pain released and total bilirubin level decreased to normal 1 month after treatment. A repeat CT scan was performed after treatment and the pancreatic lesion was disappeared (Figure 2. D).

Pancreatic tuberculosis is extremely rare. Common clinical manifestations of pancreatic tuberculosis include abdominal pain, fever, anorexia, back pain, jaundice and weight loss (1). But, pancreatic cancer also has the same clinical features. On imaging, pancreatic tuberculosis is usually characterized as a cyst-solid or multicystic mass (2). It is difficult to distinguish between pancreatic tuberculosis and pancreatic tumor. EUS-FNA can make a definite diagnosis before operation. In the present case,
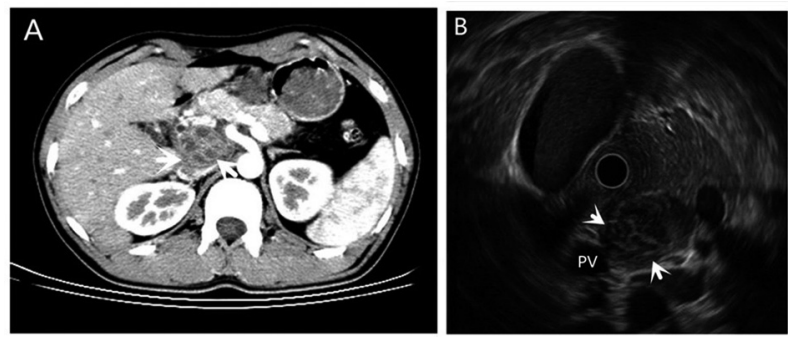

Figure 1. - (A) Abdominal contrast-enhanced CT showed a 25 x $30 \mathrm{~mm}$ cyst-solid mass in the head of pancreas (arrows). (B) Endoscopic ultrasound confirmed the mass with uneven echo in the pancreatic head and the lesion may invade the portal vein (arrows).
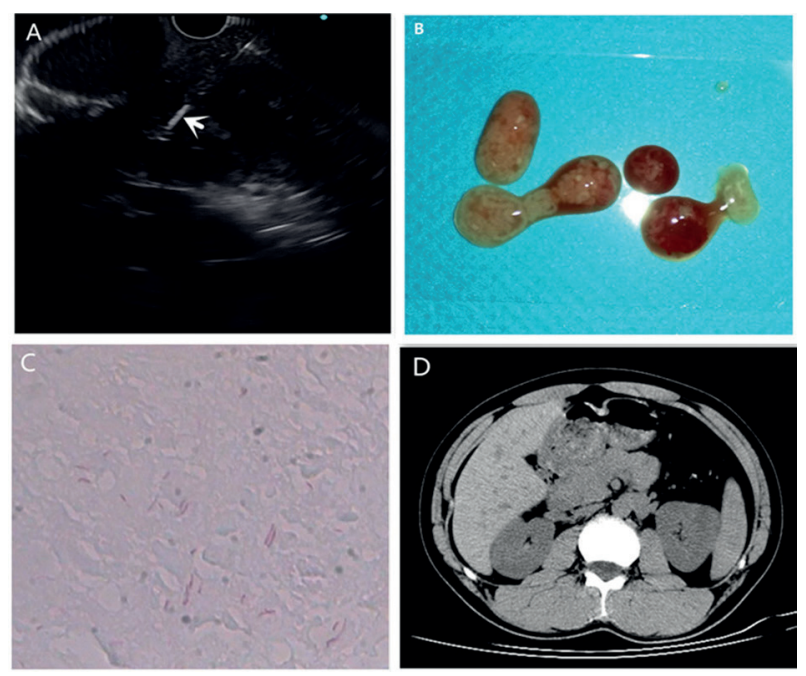

Figure 2 - (A) EUS-FNA of the lesion was performed (arrow). (B) Some specimen acquired from EUS-FNA was caseous. (C) Mycobacterium complex was found in acid-fast bacillus smear. (D) Repeat CT scan was performed after treatment and the pancreatic lesion was disappeared.

EUS-FNA was performed in duodenum, if the lesion was a malignant tumor, the puncture route was also within the scope of surgical resection. It avoided the possibility of tumor planting metastasis. Anti-tuberculosis treatment is an effective method for pancreatic tuberculosis. ${ }^{2}$

\footnotetext{
Correspondence to: Bing Hu, M.D., Department of Gastroenterology, West China Hospital, Sichuan University, No. 37 Guo Xue Alley, Chengdu 610041, Sichuan Province, China. Phone: +86-18980601278

E-mail: hubingnj@163.com

Submission date : 20/01/2021

Acceptance date : 03/02/2021
} 
Keywords: tuberculosis, cyst-solid mass.

\section{Financial Disclosure}

All authors have no financial relationships relevant to this article to disclose.

\section{Conflict of interest}

All authors have no conflicts of interest to disclose.

\section{References}

1 WOODFIELD JC, WINDSOR JA, GODFREY CC et al. Diagnosis and management of isolated pancreatic tuberculosis: recent experience and literature review. ANA J Surg 2004; 74: 368-371.

2 SHARMA V, RANA SS, KUMAR A et al. Pancreatic tuberculosis. $J$ Gastroenterol Hepatol 2016; 31: 310-318. 